\title{
DINÂMICA INTERACIONAL DE CRIANÇAS EM GRUPO: UM ENSAIO DE CATEGORIZACÇÃO
}

\author{
Ana Maria Almeida Carvalho* \\ Ângela Uchoa A. Branco" \\ Maria Isabel Pedrosa" \\ Maria Stella C. de A. Gil
}

\begin{abstract}
RESUMO. O presente estudo teve por objetivo descrever o fluxo de interações de crianças de uma mesma idade em uma situação de brinquedo. Foi registrada, durante 30 minutos, através de videotape, uma situação lúdica, semi-estruturada, onde quatro crianças de três anos brincavam livremente com objetos variados. Foram escolhidos arbitrariamente cinco minutos para análise, e elaboradas quatro categorias de interação a partir dos atributos de regulação e orientação da atenção das crianças. Estas categorias foram representadas graficamente e permitiram a elaboração de diagramas seqüenciais, possibilitando a captação do fluxo interacional. As características metodológicas desta proposta de categorização foram analisadas em comparação com as de outros trabalhos que descrevem a interação social e foram apontadas sua utilidade e limitações potenciais.
\end{abstract}

Palavras-chave: interação social, categorização, registro em vídeo.

\section{INTERACTIONAL DYNAMICS IN A GROUP OF CHILDREN: ESSAYING ITS CATEGORIZATION}

\begin{abstract}
The present study aimed at the description of the interactional flow among same-aged children during play. A loosely structured free play situation with four 3-year-old children was videorecorded during 30 minutes. A period of 5 minutes was arbitrarily chosen for analysis. Four categories were defined, based on criteria of regulation and orientation of attention by the children. The categories were represented in graphs in order to elaborate sequence diagrams which allowed the description of the interactional flow. The methodological characteristics of this system of categories were analyzed in relation to other studies on social interaction and its potential usefulness and limitations were pointed out.
\end{abstract}

Key words: social interaction, categorization, videorecording.

As interações que ocorrem entre crianças pequenas têm sido objeto de crescente interesse nas últimas décadas. Um desafio comum enfrentado pelos pesquisadores da interação social refere-se às opções metodológicas envolvidas na sua descrição. Esse desafio apresenta dificuldades peculiares no caso da interação de crianças uma vez que estas são freqüentemente observadas em grupo. Esta situação é mais complexa, do ponto de vista descritivo, do que a situação diádica que caracteriza muitos estudos de interação mãecriança (Schaffer, 1977).
Na ocasião em que este trabalho foi originalmente realizado (meados da década de 80), a área de estudos de interação social encontrava-se particularmente efervescente em termos metodológicos e conceituais, espelhando um momento de transição entre abordagens mais descritivas e estritamente comportamentais e inquietações teóricas a respeito da adequação dessas abordagens à compreensão da dinâmica interacional e de processos sociais. Nesse contexto, o ensaio de categorização aqui apresentado apontou um caminho de pesquisa que, ao longo da última década, resultou em várias outras publicações,

\footnotetext{
Universidade de São Paulo.

Endereço para correspondência: Rua da Invernada, 12, Fazendinha - Carapicuíba, SP - 06355-340. E-mail: amacarva@uol.com.br

\# Universidade de Brasília.

II Universidade Federal de Pernambuco.

æ Universidade Federal de São Carlos.
} 
centradas na conceituação e na análise de interações sociais, conduzindo a uma abordagem dos fenômenos interacionais mais processual e mais consistente em termos teóricos, como ilustram diversos trabalhos posteriores, e.g. Branco (1998), Branco e Junqueira (1993), Branco, Parro, Costa e Queiroga (1996), Carvalho (1992), Carvalho, Império-Hamburger e Pedrosa (1998), Gil e Almeida (2000), Pedrosa, Carvalho e Império-Hamburger (1997). O presente texto, embora revisado, não representa o estado atual desses desenvolvimentos. Em nossa avaliação, não seria cabível nem válido retomar a proposta original para reformulá-la nesse sentido. Seu valor, a nosso ver, é justamente o de constituir um ponto de partida, um momento temporalmente situado da reflexão sobre processos interacionais. É o acesso a esse momento que julgamos justificável oferecer através de uma republicação ${ }^{1}$.

Hinde (1979) enfatiza a importância de uma firme base descritiva para o estudo do comportamento social e sugere que a confusão conceitual que caracteriza o estudo das relações sociais seja devida, pelo menos parcialmente, à inexistência de um "mapa descritivo" que oriente a pesquisa paradigmática e permita a avaliação de seus resultados. Como contribuição à descrição e análise do comportamento social, Hinde e Stevenson-Hinde (1976) propõem um quadro conceitual em que o comportamento social é situado em três níveis: interações, relações e estrutura social.

Para descrever uma interação é necessário especificar o que os participantes estão fazendo juntos (conteúdo) e como o fazem (qualidade). A descrição de uma relação envolve a descrição do conteúdo e qualidade das interações componentes e também de sua padronização no tempo. A descrição da estrutura social requer a descrição do conteúdo, da qualidade e da padronização das relações que a constituem (Hinde e Stevenson-Hinde, 1976, p. 451, grifos nossos).

Assim, por exemplo, num grupo de macacos rhesus, a partir da descrição de casos particulares de interação (interações de amamentação entre mãe A e filho B, interações de limpeza entre A e B, interações lúdicas entre $\mathrm{A}$ e $\mathrm{B}$, etc.) são abstraídas as características que definem essas várias modalidades de interação nessa díade. O mesmo se dá para outras díades mãe-filho, bem como para outros tipos de parceiros (interações de limpeza entre fêmea A e

\footnotetext{
O exemplar da revista onde o artigo foi publicado não circulou entre a comunidade científica, tendo sido suspenso o trabalho gráfico do referido exemplar por inviabilidade financeira.
}

macho $\mathrm{C}$, interações de cópula entre $\mathrm{A}$ e $\mathrm{C}$, etc.). Num segundo nível, definem-se as relações mãe-filho, macho-fêmea, etc. pelos tipos de interações que as constituem e sua padronização: relação mãe-filho constituída por interações de amamentação, limpeza, transporte, brincadeira, etc., com determinadas qualidade e padronização própria; relação machofêmea, constituída por interações de limpeza social, de cópula, etc., também com qualidades e padronização características; e assim, sucessivamente, para todos os tipos de relações identificados na situação. Finalmente, a estrutura do grupo é definida pelas relações entre seus membros e a padronização destas, o que permite diferenciá-lo de outros grupos (Hinde e Stenvenson-Hinde, 1976).

Este esquema conceitual, sem dúvida útil como referencial para o estudo empírico e a ordenação de fatos referentes ao comportamento social, avança também a questão da descrição da interação social, ao diferenciar duas "dimensões" descritivas: conteúdo e qualidade.

Por outro lado, em qualquer das dimensões, um problema central que se coloca é o dos critérios de recorte de segmentos específicos do fluxo interacional. Como a maior parte dos eventos comportamentais, o evento interativo, qualquer que seja sua definição, não se apresenta, tipicamente, de forma discreta no tempo e no espaço, mas sim imerso num fluxo de eventos, cuja descrição exige algum tipo de recorte. Neste nível emergem simultaneamente problemas que dizem respeito à amplitude das unidades de análise a serem adotadas, e problemas relativos à captação do fluxo de interações.

Podem-se identificar, portanto, pelo menos três níveis de considerações metodológicas relativas à descrição da interação social: dimensões descritivas, unidades de análise (categorias) e captação do fluxo de eventos no qual as unidades estão imersas.

Os problemas referentes à amplitude das unidades de análise e à captação do fluxo de interações têm sido extensamente discutidos na literatura da área. É interessante notar neste contexto que, em geral, as discussões abordam isoladamente essas questões, não as situando umas em relação às outras. Assim, por exemplo, diversos autores apontam para o fato de que as tentativas de descrever a interação social frequientemente têm se constituído em decomposição de seqüências em unidades elementares, cujo conjunto não mais permite a captação do processo interativo que está ocorrendo entre os indivíduos envolvidos (Blurton-Jones, 1981; Richards, 1974). Buscando comparar o valor metodológico de diversos tipos de recorte de seqüências interativas na relação mãecriança, Lewis e Lee-Painter (1974) salientam a necessidade de contextualizar a interação e considerála como um fluxo contínuo de eventos. 
A análise de sequiências talvez seja o procedimento mais conhecido e elaborado de análise de dados observacionais com o objetivo de descrever o fluxo dos eventos ou suas relações temporais. Bakeman e Gottman (1986) dão ênfase ao fato de que uma característica definidora da interação é que ela se desenrola no tempo e, na verdade, mal pode ser pensada sem uma referência temporal. Diante disso, esses autores consideram os métodos de Análise Sequiencial especialmente adequados para o estudo de interações sociais, embora sejam aplicáveis também a outros tipos de seqüências comportamentais.

A amplitude da unidade de análise também tem sido bastante focalizada na literatura. Diversos aspectos dessa questão são explorados: a utilidade de categorias moleculares versus molares; critérios subjacentes à definição de categorias (morfologia, função, fatores causais comuns); o uso de padrões interativos como unidades, etc. (Lamb e Sherrod, 1981).

Uma contribuição para essa questão é oferecida por Camaioni (1980, p. 29) na proposta de uma categorização em que as unidades de análise são “... não os comportamentos do indivíduo, mas os comportamentos de interação, os quais são, por definição, eventos interindividuais mais que intraindividuais (...) a unidade mínima é o evento diádico". As categorias de interação propostas por Camaioni são definidas a partir de relações entre os comportamentos dos parceiros: contemporaneidade versus alternância; similaridade versus complementaridade; revezamento de papéis. Camaioni distingue ainda, entre essas relações, as que se referem a aspectos estruturais da interação (contemporaneidade/alternância e revezamento) e as que se referem a aspectos de conteúdo (complementaridade/similaridade). Esta distinção introduz novamente a questão das dimensões descritivas, já apontada acima, acrescentando às dimensões conteúdo e qualidade, propostas por Hinde e Stevenson-Hinde (1976), a noção de estrutura da interação. Para Camaioni, estrutura refere-se à organização seqüencial do comportamento e não ao que aqueles autores chamaram de estrutura social quando trataram dos três níveis possíveis na descrição e análise do comportamento social.

Deve-se observar que, embora a organização temporal da interação seja levada em conta na proposta de Camaioni, ela se refere às relações temporais entre os comportamentos dos parceiros em um evento interativo definido por um comportamento de cada parceiro (uma iniciativa/uma resposta). A descrição do encadeamento ou fluxo de interações não é abordada nesse trabalho.
A questão das relações temporais na descrição da interação social levanta perguntas como: o que caracteriza o fluxo de interações? Que problemas essa ou essas características colocam para a descrição do comportamento social?

Dentro dessa problemática foi proposto este trabalho. O objetivo foi refletir sobre a descrição da interação de crianças e experimentar a elaboração de uma nova proposta. A questão principal estava relacionada à possibilidade de captar o fluxo temporal das interações. Conseqüentemente, definições do tipo de recorte e das unidades de análise a serem adotadas deveriam decorrer dessa questão.

Com esse objetivo planejou-se uma situação para observar crianças interagindo. Em consonância com o objetivo do trabalho, unidades comportamentais não foram assumidas a priori; foram definidas à medida que eram identificadas regularidades na seqüência de interações observadas. A opção pela coleta de dados através de registro em vídeo derivou, portanto, desta proposta de trabalho. As demais opções relativas à coleta de dados foram tomadas visando maximizar a probabilidade de ocorrência de eventos interativos que servissem de material para análise e reflexão. Os registros foram realizados em uma situação lúdica semi-estruturada, onde quatro crianças que se conheciam mutuamente tinham a oportunidade de brincar livremente com objetos variados, num ambiente familiar e sem a presença da professora. A opção por um grupo, de preferência a uma díade, foi encorajada pelo desafio que representa a descrição do comportamento social numa situação mais complexa e mais semelhantes à usual nos estudos naturalísticos sobre interação de crianças.

\section{MÉTODO}

\section{Sujeitos}

Quatro crianças, dois meninos e duas meninas, cujas idades variavam de três anos a três anos e um mês, foram selecionadas, arbitrariamente, de um grupo de oito crianças que conviviam havia cerca de dois anos e meio em uma creche pública da cidade de São Paulo $^{2}$.

\section{Condições de observação}

O registro de observação realizou-se na creche, em uma sala previamente organizada pelos observadores. Para captar melhor a fala das crianças,

\footnotetext{
2 Agradecemos à direção da Creche da Universidade de São Paulo por ter permitido a observação e filmagem das crianças.
} 
um microfone foi pendurado no teto, sobre a mesa. $\mathrm{O}$ mobiliário da sala foi disposto de modo a delimitar uma área de aproximadamente $64 \mathrm{~m}^{2}$. Uma mesa baixa foi colocada no centro desta área. Sobre a mesa ficaram, à disposição das crianças, vários brinquedos manufaturados (como miniaturas de bonecos, vagões de trem, carros de boi, etc.); material de encaixe (peças de diferentes formas) e outros objetos como óculos, latas vazias, etc.

O registro foi realizado em uma única sessão, durante 30 minutos. As quatro crianças foram levadas à sala por um dos três observadores que permaneceram na sala durante o registro. As crianças brincaram sem a participação dos observadores.

\section{Procedimento de tratamento dos dados}

Para a seleção e delimitação de um trecho do registro, os observadores assistiram diversas vezes à sessão completa. Tendo em vista o caráter exploratório do trabalho, foi estabelecido que apenas uma parte do registro seria selecionada para tratamento e análise.

Revendo diversas vezes a gravação, alguns momentos pareciam mais conspicuamente interativos do que outros. Nesses momentos percebiam-se, por exemplo, duas ou mais crianças orientadas mutuamente, compartilhando os mesmos brinquedos, conversando sobre um tema, disputando um objeto, etc. Arbitrariamente foi escolhido um desses momentos para ser analisado, com duração total de cinco minutos.

Para a transcrição dos dados a seguinte sistemática foi obedecida: num primeiro momento foram descritos os comportamentos de cada criança isoladamente, na ordem temporal de ocorrência. Nessa descrição relatavam-se a verbalização, a postura, a movimentação, o brinquedo manipulado e, se possível, a direção do olhar. Dessa forma foram obtidos quatro protocolos de transcrição, chamados de protocolos $\mathrm{P} 1$, referentes a cada um dos sujeitos.

Num segundo momento foram compatibilizados os comportamentos das quatro crianças simultaneamente em um único protocolo, sincronizando os eventos transcritos. Para isso, os observadores voltavam a assistir à gravação e procediam da seguinte maneira: indicavam o comportamento de uma criança e assinalavam os das outras três que aconteciam na mesma ocasião. Construiu-se, desse modo, um segundo protocolo (P2) com os comportamentos sincronizados das quatro crianças. A elaboração das categorias e sua utilização para a análise dos dados foram realizadas através da leitura repetida de $\mathrm{P} 2$, acompanhada de observação do vídeo no trecho selecionado.

\section{RESULTADOS}

\section{Elaboração das categorias e suas codificações}

A elaboração das categorias foi orientada pelo objetivo de descrever o fluxo interacional, entendido como o movimento ou dinâmica dos contatos sociais no grupo ao longo do tempo. A descrição deveria incluir, tanto os momentos em que um ou mais parceiros estabelecem intercâmbios conspícuos como aquelas ocasiões em que os contatos parecem se desfazer, e as crianças apresentam comportamentos predominantemente voltados para suas próprias atividades. As categorias elaboradas com esse intuito foram baseadas em dois atributos, quais sejam: a orientação da atenção das crianças e o tipo de regulação envolvido entre elas. Identifica-se orientação quando se detecta o alvo das ações da criança através do olhar e/ou da fala e/ou do movimento. Identifica-se regulação quando se detecta, através do conteúdo do comportamento dos sujeitos (movimento, fala, uso de objetos, etc.), efeito das ações de uma criança sobre outra, ainda que não se possa precisar que aspectos das ações de uma atingiram a outra. A combinação desses atributos resultou no sistema de categorias descrito a seguir. Nos exemplos apresentados, a notação " / " significa comportamentos seqüenciados; a notação " ! " significa concomitância de comportamentos. As letras A, B, C, e D correspondem às crianças observadas.

I - Interação: quando as crianças se orientam mutuamente, havendo regulação recíproca caracterizada pela complementaridade conspícua de ações.

\section{Exemplo:}

A fala "cadê, cadê" / olha mesa varrendo um ângulo de $180^{\circ}$.

C coloca as mãos sobre a mesa (uma das mãos cobre uma galinha).

B olha em direção à mesa / fala "num tá ... onde tá a outra galinha?"

A olha em diferentes direções.

B fala “... tá com ela, ó ..."; aponta na direção da mesa em frente a $C$.

C olha B / larga a galinha sobre a mesa.

Neste exemplo, as crianças A, B e C estão em interação.

MI - Movimento Interativo: quando uma criança se orienta para outra, havendo uma regulação recíproca porém restrita, implicando na 
reorientação da atenção da criança-alvo para outros objetos, parceiros ou atividades.

Exemplo:

C pega carrinho / coloca carrinho à sua frente.

A estende a mão em direção a $\mathrm{C} /$ pega carrinho da mão de C / fala "deixa eu pegar isso" / segura o carrinho com as duas mãos.

C solta carrinho / pega pás.

A coloca o carrinho sobre a mesa à sua frente.

Neste exemplo, as crianças A e C estão em MI; a criança C é o alvo do MI.

PP - Participação Periférica: quando uma criança se orienta para outra, havendo uma regulação unilateral, isto é, a criança-alvo regula o comportamento da primeira mas mantém sua orientação prévia, sem ser regulada por esta.

Exemplo:

B segura com a mão esquerda duas pás formando um " L " / ergue seu braço esquerdo / olha em direção às pás / fala "oooooonnnn, poooo, po"

$\mathrm{D}$ olha em direção a $\mathrm{B} /$ olha em direção à mesa / coloca boneco sobre a mesa / pega pás / manipula pás.

B pega carrinho.

Neste exemplo a criança D está em PP; a criança B é o alvo de sua orientação.

AI - Atividade Individual: quando uma criança se orienta para um brinquedo ou atividade que se desenrola sem que se detecte regulação de qualquer outra criança sobre ela.

\section{Exemplo:}

A encaixa pino no carrinho / fala "tchft" / anda / apóia duas mãos sobre a mesa / saltita.

Após a elaboração das categorias foram criados códigos que permitiram representar graficamente as relações e o movimento do grupo no contínuo analisado. Essa representação teve por objetivo facilitar a captação do fluxo interativo, pois este, facilmente apreendido através do vídeo, dificilmente era percebido num relato escrito. A codificação permitiu também a indicação da criança-alvo, ou seja, aquela que é objeto da atenção da criança que inicia o intercâmbio no movimento interativo e na participação periférica. Essa codificação está indicada na legenda da Figura 1 , como veremos mais adiante.
Categorização dos comportamentos e descrição do fluxo

Definidas as categorias retomou-se o P2, passando-se a relacionar os diferentes tipos de intercâmbio existentes entre as crianças ao rol de categorias. Obteve-se assim a categorização dos comportamentos transcritos.

É importante enfatizar o caráter relacional dessas categorias. Constituídas a partir dos atributos de regulação e orientação da atenção, elas retratam a influência de uma criança sobre outra(s) e a direção de seus comportamentos. Assim, a indicação de que uma criança se encontra em uma determinada categoria implica pensar um tipo de relação desta criança com outra(s). Trata-se, portanto, de categorias interindividuais. A categoria I (Interação), por exemplo, implica mútua regulação e mútua orientação. Informando-se que a criança está em interação, forçosamente se está afirmando que existe, no grupo, pelo menos uma outra também em interação. Observese que a AI (Atividade individual), curiosamente, também indica o comportamento da criança em relação ao grupo, porque informa que ela está, naquele momento, com sua atenção voltada predominantemente para sua própria atividade ou brinquedo, sem que se detectem no grupo indícios de regulação de outra criança sobre ela.

As categorias possibilitam, por outro lado, pensar em diversas relações envolvendo a mesma criança em um dado momento. Deste modo, por exemplo, uma criança pode estar em AI mas regulando o comportamento de outra, ou seja, ser criança-alvo de PP (Participação Periférica) dessa outra. Ou ainda, uma criança em AI pode ser alvo de MI (Movimento Interativo) de uma terceira criança do grupo.

Essas características, embora necessárias à descrição do fluxo de interação, não a asseguravam. Fazia-se necessário, ainda, assinalar as alterações ocorridas no tipo de intercâmbio mantido entre as crianças, preservando-se a ordem em que ocorriam. Decidiu-se então construir diagramas seqüenciais que retratassem essas alterações. A construção de cada novo diagrama baseou-se na alteração da natureza dos intercâmbios entre os sujeitos, bastando que uma criança modificasse seu comportamento em relação ao grupo para que se acrescentasse outro diagrama à sequiência. Vale salientar, contudo, que os diagramas não representam a mesma fração de tempo, ou seja, eles podem corresponder, por exemplo, a 3,4 ou 6 segundos se for essa a duração dos intercâmbios representados naqueles diagramas. 
Na construção da sequiência de diagramas foram adotados os seguintes critérios: a ordem de ocorrência dos eventos no tempo e a alteração da natureza dos intercâmbios entre os sujeitos. Obteve-se, com esses critérios, uma seqüência de 40 diagramas, apresentada no Figura 1.

\begin{tabular}{|c|c|c|c|c|c|c|}
\hline 1 & A $\quad$ B & B & (C) & 21 & $A 4 \cdots B$ & C $\cdots \cdots$ \\
\hline 2 & A 4 (E & B $4-1$ & $\mathrm{C}$ & 22 & $\mathrm{~B} \triangleleft \mathrm{C}$ & C \\
\hline 3 & A 4 & B $4+\cdots$ & c & 23 & $B \stackrel{\longrightarrow}{\hookrightarrow} C$ & $C \quad D$ \\
\hline 4 & $A \longleftrightarrow$ & B & (C) D & 24 & A & C $4 \cdots D$ \\
\hline 5 & $A \stackrel{\longrightarrow}{\longrightarrow}$ & B & C & 25 & A $4 \cdots B$ & C $4 \cdots D$ \\
\hline 6 & $A \stackrel{\longrightarrow}{\longrightarrow} B$ & B & $\mathrm{C}$ & 26 & A $4 \cdots B$ & $C \rightarrow D$ \\
\hline 7 & $A \stackrel{\forall}{\leftrightarrow}$ & B & D & 27 & $A \underset{\Delta}{\longrightarrow} B$ & $C \quad \ldots \rightarrow D$ \\
\hline 8 & $A \stackrel{b}{\longrightarrow}$ & & (C) D & 28 & $A \stackrel{\vec{\Delta}}{\longrightarrow} B$ & $C \quad D$ \\
\hline 9 & A $\mathrm{E}$ & $B$ & (C) $\rightarrow D$ & 29 & ${ }_{\Delta}^{A} \quad B_{\Delta}^{B}{ }_{\Delta}$ & $\mathrm{C}$ \\
\hline 10 & A & $B$ & $C \quad D D$ & 30 & $\begin{array}{lll}A & B & C \\
\Delta & C\end{array}$ & $\begin{array}{ll}C & D \\
\end{array}$ \\
\hline 11 & A & $B^{4 \cdots}$ & C $\rightarrow D$ & 31 & $A \underset{\Delta}{\longrightarrow} B$ & C \\
\hline 12 & $A_{4} \ldots$ & $B^{4-\cdots}$ & $C$ & 32 & $A \leftrightarrow \stackrel{\forall}{\leftrightarrow} B \quad C$ & C \\
\hline 13 & A & B & $C \cdots \cdots D(D$ & 33 & $A \stackrel{\forall}{\Delta} B$ & $\mathrm{c}$ \\
\hline 14 & A $4 . .$. & B & $\mathrm{C}$ & 34 & $A \stackrel{b B}{\longleftrightarrow}$ & C \\
\hline 15 & A & B & $C \cdots \cdots D$ & 35 & $A+\cdots B_{4} 4 \ldots D_{C}$ & $C$ \\
\hline 16 & A & B & (C) & 36 & $A \cdots B_{\triangleleft} \leftrightarrow D_{C} C$ & C \\
\hline 17 & A & $B_{4} \ldots$ & C & 37 & $A \leadsto B$ & C D \\
\hline 18 & $\underset{\Delta}{A}$ & $\mathrm{~B}^{4+3}$ & $\underset{\triangle}{C}$ & 38 & A $\cdots B_{4}$ & $C \quad \cdots D$ \\
\hline 19 & $A$ & $\mathrm{~B} \longleftrightarrow$ & C & 39 & $A \stackrel{\square}{\Delta} B$ & $C 4 \cdots D$ \\
\hline 20 & A & B & (C) & 40 & $\begin{array}{lll}A & B \\
\Delta & \nabla & \Delta \\
\end{array}$ & $C_{\Delta} \quad D$ \\
\hline $\mathrm{A}, \mathrm{B}, \mathrm{C}, \mathrm{D}$ & $\begin{array}{l}\text { CRIANCC, } \\
\text { INTERAC } \\
\text { MOVIME }\end{array}$ & $\begin{array}{l}\text { ÇAS } \\
\text { CČ̃̃O } \\
\text { ENTO INTE }\end{array}$ & IERATIVO & & $\begin{array}{l}\text { PARTICIPAČ̃̃ PER } \\
\text { ATIVIDADE INDIVID } \\
\text { FORA DO CAMPO }\end{array}$ & $\begin{array}{l}\text { RIFÉRICA } \\
\text { IDUAL }\end{array}$ \\
\hline
\end{tabular}

Figura 1. Diagramas de intercâmbio em sua seqüência de ocorrência

Os 40 diagramas (5 minutos de registro) observados em sua sequiência permitem a captação do fluxo interativo através do movimento e da dinâmica do grupo aí representados.

Observando-se os diagramas ordenados no tempo vê-se como se dão os comportamentos das crianças umas em relação às outras fluem, percebendo-se a "polarização" da atenção do grupo, ora para um lado (criança localizada graficamente em um dos lados do diagrama), ora para o outro. Esses pólos de atenção são indicados pelas direções das setas que fazem parte da notação para simbolizar as categorias.
Nos três primeiros diagramas aparecem PP e MI, sendo as crianças A e B o alvo dos intercâmbios. Do diagrama 4 ao 8 acontecem interações das crianças $A$ e $\mathrm{B}$, enquanto as duas outras estão orientadas para aquela díade, em PP. Desta forma, a orientação do grupo volta-se para o lado esquerdo do diagrama, ou seja, para as crianças A e B.

A partir do diagrama 9 a criança A entra em AI e as outras reorientam-se mutuamente. A criança D começa a ser alvo de atenção e passa a regular também o comportamento do grupo. A partir do diagrama 12 a criança B também entra em AI, e esta forma de intercâmbio predomina no grupo até o diagrama 17, embora ocorram dois $\mathrm{MI}$ de $\mathrm{C}$ para $\mathrm{D}$ e algumas PP destas duas crianças em relação às outras. Este trecho antecede as I representadas nos diagramas 18 e 19 e se diferencia de outros que também antecedem sequiências interativas, tais como os trechos que vão do 25 ao 26, do 35 ao 38, e ainda aquele já comentado, os diagramas de 1 a 3 . No trecho de 35 a 38, por exemplo, a PP é a forma de intercâmbio dominante, que parece preparar a ocorrência de I, enquanto no trecho de 12 a 17 a I aparece em seguida a uma situação de AI predominante.

Confrontando-se os trechos com predomínio de PP que antecedem seqüências interativas, nota-se que: no primeiro deles, dos diagramas 1 a 3, a orientação do grupo se volta para uma criança (A) que entra em I em seguida. No segundo trecho, do 25 ao 26, não se pode falar de uma criança privilegiada como alvo dos intercâmbios. O que chama a atenção nos registros é uma tendência de as crianças se orientarem em pares. No terceiro trecho, que vai do 35 ao 38, mesmo com a atenção do grupo voltando-se para uma criança (B), existem intercâmbios entre as outras que aumentam a complexidade da rede de relações do grupo. A criança (B), alvo de atenção, entra, em seguida, em I.

Em relação ao tipo de intercâmbio ocorrido, observa-se o predomínio da PP como forma de intercâmbio (vê-se em 31 diagramas), seguida de I (19 diagramas) e finalmente de MI (9diagramas). A PP tem como alvo a I de duas ou mais crianças em 15 diagramas, enquanto que as crianças que estão em AI são alvo dela em 10 diagramas. Apenas em três deles (4, 19 e 22) a I não é acompanhada de PP. Quando se comparam os diagramas em que ocorrem simultaneamente I, PP e AI $(6,8,23,31,34)$, nota-se que não há caso de PP tendo como alvo a criança que está em AI.

Verifica-se, ainda, que as interações ocorrem predominantemente entre as crianças $\mathrm{A}$ e B, envolvendo a criança $\mathrm{C}$ duas vezes $(29,40)$ e apenas uma vez as quatro crianças (30). Por outro lado, as 
crianças C e D envolvem-se principalmente em PP. É interessante perceber que a criança $\mathrm{B}$ parece tecer mais as relações do grupo: ela interage preferencialmente com a criança $\mathrm{A}$, seguida da $\mathrm{C}$, ao mesmo tempo em que participa perifericamente e é alvo de PP por parte das crianças C e D. É interessante também observar que em apenas três diagramas $(9,10$, 11), excluindo-se aquele onde todos estão interagindo, a criança $\mathrm{D}$ orienta-se para um parceiro que de alguma forma esteja se relacionando com ela.

A Figura 2 representa uma leitura dos diagramas, criança por criança. Para a elaboração dessa figura, as categorias foram indicadas na ordenada de acordo com o grau crescente de envolvimento social ou de regulação mútua que está implicado em suas definições. Os diagramas, dispostos na abcissa, são tratados como uma variável contínua. Isso se justifica considerando-se que, embora não se baseiem em uma divisão convencional do tempo (minutos ou segundos), eles representam o contínuo do tempo, subdividido por um critério arbitrário mas constante para os quatro membros do grupo (cada ponto representa o mesmo minuto para as quatro crianças). Comparando-se os traçados relativos às crianças, vê-se a possibilidade de agrupá-los dois a dois pela semelhança que apresentam entre si. Os traçados A e B (meninos) aproximam-se por retratarem as crianças na mesma categoria, na mesma ocasião; enquanto os traçados das crianças C e D (meninas) assemelham-se porque retratam uma variabilidade de intercâmbios dessas crianças no mesmo período de tempo.

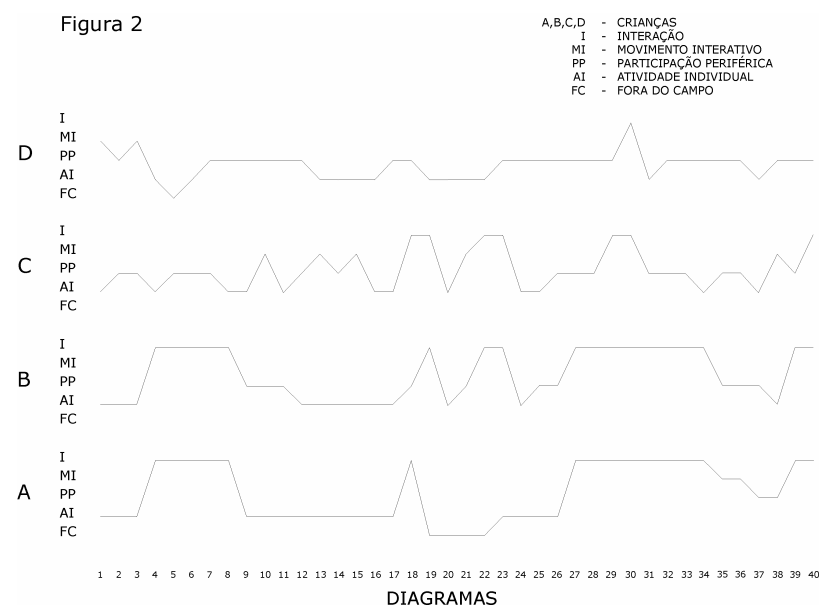

Figura 2. Fluxo de interações, por criança.

As crianças A e B, de modo geral, alternaram AI e I. Elas diferem entre si pelo envolvimento da criança A em MI e pelo maior número de envolvimentos da criança B em PP.

O traçado da criança C caracteriza-se por uma grande variabilidade do tipo de intercâmbio. Quanto à criança D, predomina a PP como forma de relacionarse nesse grupo. Ocorre apenas um envolvimento interacional.

\section{DISCUSSÃO}

De que forma se situa esta proposta em relação aos três níveis de consideração metodológica relativos à interação social que foram levantados na introdução deste trabalho?

Quanto à dimensão descritiva, esta proposta privilegia a estrutura das interações, ou seja, o tipo de relação entre os comportamentos das crianças que caracteriza o grupo em cada momento. Neste sentido, assemelha-se à categorização de Camaioni (1980). Por outro lado, diferencia-se desta quanto à natureza das relações que servem de base à categorização. No caso de Camaioni, dois tipos de relações são considerados: relação temporal entre os comportamentos das crianças (simultâneos ou não), e a relação de semelhança (ou não) de conteúdo das ações. A dimensão conteúdo (o que os parceiros fazem) é portanto utilizada, mas apenas como subsídio para a definição da relação de semelhança, e não como critério direto de categorização. Da mesma forma, na presente proposta o conteúdo é considerado para subsidiar a identificação de relações entre os comportamentos das crianças. As relações consideradas são, no entanto, de natureza diferente das de Camaioni: são as relações de orientação e regulação que subjazem à descrição estrutural da interação no caso deste trabalho.

Esta diferença de critério dentro de um mesmo tipo de opção em termos de dimensão descritiva resulta numa diferença interessante quanto ao que é descrito por esses dois sistemas de categorias estruturais. As categorias de Camaioni (1980) aplicam-se apenas aos eventos que, no presente trabalho, foram denominados interações. Para aquela autora, a orientação é tomada como pré-requisito para a ocorrência de interação e esta, por definição, envolve regulação mútua; os casos em que o comportamento de um parceiro não é regulado pelo do outro são tratados como interações fracassadas ou inadequadas. Esta opção elimina do conjunto de informações colhidas as aproximações e afastamentos que ocorrem entre os membros de um grupo. São esses movimentos, justamente, que permitem acompanhar as modificações do intercâmbio interpessoal que conduzem ou não para a efetivação das trocas comportamentais denominadas interações, e assim captar o que foi chamado, neste trabalho, de fluxo ou dinâmica de interações sociais. Desta forma, 
o que o sistema de categorias apresentado neste trabalho procura descrever é um universo de eventos mais amplo do que o focalizado no sistema de Camaioni, o qual inclui este último: um universo que vai da AI à I propriamente dita, e no qual a AI também é tratada como um evento de comportamento social, pelo fato de informar que a atenção dessa criança está voltada para sua própria atividade ou brinquedo e que não se detectam, no grupo, indícios de regulação de outra criança sobre ela. Seria possível, portanto, superpor os dois sistemas de categorias, ou seja, utilizar o sistema de Camaioni para sub-categorizar as instâncias de I identificadas pelos critérios propostos aqui ${ }^{3}$. Por outro lado, aquele sistema não se aplica às outras categorias aqui descritas, nem as substitui. Evidentemente, seria possível também superpor a este sistema um outro, baseado em outra dimensão (conteúdo e/ou qualidade) se os objetivos do trabalho o requisitassem, e desde que a pertinência e as limitações dessas superposições fossem avaliadas.

É interessante notar, portanto, que a questão das dimensões descritivas não se reduz à opção por uma determinada dimensão (estrutura, conteúdo, qualidade) ou mais de uma, mas envolve decisões a respeito dos critérios pelos quais a interação é categorizada nessa(s) dimensão(ões). No caso da dimensão estrutura, esses critérios se referem ao tipo de relação entre os indivíduos a ser considerado. No caso das demais dimensões, já se apresenta uma outra questão: a das unidades de análise. Note-se que, ao se optar pela dimensão estrutura para a descrição da interação social, alguns aspectos da questão da unidade de análise deixam necessariamente de ser opcionais: as categorias serão necessariamente interindividuais, uma vez que se baseiam em relações entre os comportamentos dos indivíduos; e a questão da amplitude da unidade (categoria) de certa forma se esvazia, uma vez que as unidades decorrerão do tipo de relação adotado como critério. O que se poderia pensar, num sistema desse tipo, é a amplitude do evento que está sendo categorizado, o que também se relaciona com a opção pelo tipo de relação a ser considerado como critério: assim, poder-se-ia dizer que o sistema de categorização aqui proposto se baseia num recorte mais molar do que o sistema de Camaioni, uma vez que inclui o recorte deste último num sentido análogo àquele em que um sistema do tipo "Olhar para ... (X, Y, Z) é mais molar do que "Olhar de esguelha", "Olhar de relance", etc.

3 Esta abordagem foi utilizada por Carvalho (1992) para a análise de interação em díades de parceiros de brincadeira.
Uma outra característica deste sistema que deve ser salientada, e que também decorre do tipo de relação utilizado como critério de categorização, é o fato de as categorias não serem mutuamente exclusivas. Como as categorias são interindividuais, a mesma criança pode, num mesmo momento, ser classificada em PP em relação a um (alguns) parceiro(s) e ser alvo de um MI por parte de outro. Esta característica pode introduzir alguma dificuldade quando o sistema é usado para descrever o comportamento individual, como na Figura 2, mas por outro lado permite captar melhor a complexidade das relações que se estabelecem na situação de grupo.

Finalmente, resta abordar a questão da captação do fluxo de eventos no qual as unidades estão imersas, a qual foi uma preocupação central quando da proposição deste trabalho. De fato, o tipo de categorização proposto, associado à possibilidade de representação gráfica, permitiu uma descrição dos eventos categorizados em sua seqüência temporal de ocorrência, o que constitui um passo necessário para uma análise de relações entre esses eventos a partir dessa dimensão temporal. Esta análise não chegou a ser desenvolvida neste trabalho, em decorrência de duas limitações principais. Em primeiro lugar, o período total analisado (5 minutos) foi considerado muito curto e não necessariamente representativo (uma vez que foi selecionado arbitrariamente) para que se tentasse identificar, nele, padrões ou regularidades em termos do fluxo temporal de interações. Em segundo lugar, não foi feito, durante a transcrição dos dados, um registro de duração dos eventos descritos em cada diagrama, o que contribuiria para a identificação de regularidades não discerníveis com o critério de descrição utilizado. Sugere-se que o registro de durações seja incorporado à presente proposta visando ao refinamento da descrição que ela possibilita.

Convém ressaltar aqui a natureza da análise à qual se está fazendo referência. A seqüência de diagramas (Figura 1) permite pelo menos três leituras. Uma primeira é a leitura intra-diagrama, ou seja, uma leitura em que o diagrama é tomado como unidade e se buscam regularidades em termos de suas características. Esta leitura leva a constatações como a de que a I foi um alvo mais freqüente de PP do que a AI, ou de que PP foi a modalidade mais freqüente no período analisado. Uma segunda leitura é a que foi sintetizada na Figura 2, ou seja, uma leitura vertical, em que a unidade passa a ser o indivíduo, embora sempre caracterizado em relação ao grupo. A terceira possibilidade seria a leitura de conjuntos de diagramas, ou seja, uma leitura inter-diagramas. Esta 
abordagem foi esboçada na tentativa de sintetizar informações por blocos de diagramas (por exemplo, o bloco do 1 ao 3 pode ser comparado ao bloco do 12 ao 17, ou ao bloco do 35 ao 38; todos eles antecedem interações). Esta síntese constituiria o caminho para uma análise do fluxo interacional, e permitiria, eventualmente, a identificação dos ciclos ou ritmos que caracterizam o fluxo em diferentes situações, do papel das várias modalidades interacionais na dinâmica desses ciclos, etc.

Esta é a principal direção em que, sugere-se, este tipo de trabalho deveria ser retomado. Além disso, uma questão que este trabalho mantém em aberto é a do refinamento do critério de regulação, que inegavelmente requer ainda o estabelecimento de indicadores comportamentais mais precisos para sua aplicação ${ }^{4}$. Deve-se lembrar que a idéia de regulação é intrínseca ao conceito de interação e indissociável deste, e não deveria ser descartada com base nas dificuldades de definição e de estabelecimento de critérios de aplicação. Como diz Hinde (1979), é obrigação do cientista tentar elaborar instrumentos adequados ao estudo de todos os fenômenos naturais: é a importância de um problema, e não a facilidade que se encontra na sua abordagem, que justifica sua seleção para estudo.

\section{REFERÊNCIAS}

Bakeman, R. \& Gottman, J.M. (1986). Observing interaction: An introduction to seqüencial analysis. New York: Cambridge University Press.

Blurton-Jones, N. (1981). Estudos etológicos do comportamento da criança. São Paulo: Pioneira.

Branco, A.U. (1998). Cooperation, competition and related issues: A co-constructive approach. Em M. Lyra \& J. Valsiner (Eds.), Child development within culturally structured environments. Vol. 4. Construction of psychological processes in interpersonal communication (pp. 181-205). Stamford, CT: Ablex Publishing Corporation.

Branco, A.U. \& Junqueira, L. (1993). Child-child interactions within structured contexts: a mode of analysis. Em International Society for the Study of Behavioural Development (ISSBD) (Ed.), Poster Abstracts. XIIth Biennial Meetings of ISSBD (p. 26). Recife, PE: ISSBD.

Branco, A.U., Parro, C., Costa, V. \& Queiroga, V. (1996). Estudo microgenético de metacomunicação em crianças pequenas: uma contribuição metodológica [Resumos]. Em Sociedade Brasileira de Psicologia (Org.), Resumos de

4 A idéia de regulação foi retomada em vários trabalhos posteriores, por ex.: Pedrosa (1989), Pedrosa e cols. (1997), Carvalho e cols. (1998), não só como critério descritivo mas como conceito teórico. comunicações científicas. XXVI Reunião Anual de Psicologia (p. 91). Ribeirão Preto, SP: SBP.

Camaioni, L. (1980). L' interazione tra bambini. Roma: Armando Armando.

Carvalho, A. M. A. (1992). Seletividade e vínculo na interação entre crianças. Tese de Livre-docência, Instituto de Psicologia da USP, São Paulo.

Carvalho, A. M. A., Império-Hamburger, A. \& Pedrosa, M. I. (1998). Interaction, regulation and correlation in the context of human development: Conceptual discussion and empirical examples. Em M. Lyra \& J. Valsiner (Eds.), Child development within culturally structured environments. Vol. 4. Construction of psychological processes in interpersonal communication (pp. 155-180). Stamford, CT: Ablex Publishing Corporation.

Gil, M.S.C.A. \& Almeida, N.V.S. (2000). Padrões de interação entre mãe e bebê em situação de brincadeira. Cadernos de Psicologia / UFMG. 10 (1), 89-102.

Hinde, R. A. (1979). Towards undestanding relationships. New York: Academic Press.

Hinde, R.A. \& Stevenson-Hinde, J. (1976). Towards understanding relationships: Dynamic stability. Em P.P.G. Bateson e R.A. Hinde (Orgs), Growing points in Ethology (pp. 451-479). New York: Cambridge University Press.

Lamb, M.E. \& Sherrod, D. (1981). Infant social cognition. New Jersey: Erlbaum.

Lewis, M. \& Lee-Painter, S. (1974). Interactional approach to the mother-infant dyad. Em M.E. Lewis e L. Rosemblum (Eds), The effect of the infant on its caregiver (pp. 21-48). New York: John Wiley.

Pedrosa, M.I.P.C. (1989). Interação criança-criança: um lugar de construção do sujeito. Tese de Doutorado, Instituto de Psicologia da USP, São Paulo.

Pedrosa, M.I., Carvalho, A.M.A. \& Império-Hamburger, A. (1997). From disordered to ordered movement: Attractor configuration and development. Em A. Fogel, M. Lyra \& J. Valsiner (Eds.), Dynamics and indeterminism in developmental and social processes (pp. 135-151). Mahwah, NJ: LEA, Inc. Publishers.

Richards, M.P.M. (1974). First steps in becoming social. Em M.P.M. Richards (Ed), The integration of a child into a social world (pp. 83-97). New York: John Wiley.

Schaffer, H.R. (1977). Studies in mother-infant interaction. New York: Academic Press.
Recebido em 10/07/2002

Revisado em 22/10/2002 Aceito em 30/10/2002 
